\title{
Acquired Fanconi Syndrome with Proximal Tubular Cytoplasmic Fibrillary Inclusions of $\lambda$ Light Chain Restriction
}

\author{
Ying Yao, Su-Xia Wang, You-Kang Zhang, Yan Wang, Li Liu and Gang Liu
}

\begin{abstract}
Light chain proximal tubulopathy is a rarely reported entity associated with plasma cell dyscrasia that classically manifests as acquired Fanconi syndrome and is characterized by the presence of $\kappa$-restricted crystals in the proximal tubular cytoplasm. We herein present a case of multiple myeloma with Fanconi syndrome and acute kidney injury due to light chain proximal tubulopathy with light chain cast nephropathy. Prominent phagolysosomes and numerous irregularly shaped inclusions with a fibrillary matrix in the cytoplasm of the proximal tubules were identified on electron microscopy. A monotypic light chain of the $\lambda$ type was detected in the distal tubular casts, proximal tubular cytoplasmic lysosomes and fibrillary inclusions on immunofluorescence and immune electron microscopy. This case underscores the importance of conducting careful ultrastructural investigations and immunocytologic examinations of light chains for detecting and diagnosing light chain proximal tubulopathy.
\end{abstract}

Key words: Fanconi syndrome, immunoglobulin light chains, multiple myeloma

(Intern Med 53: 121-124, 2014)

(DOI: 10.2169/internalmedicine.53.0836)

\section{Introduction}

Renal diseases associated with plasma cell dyscrasia (PCD) most frequently include light chain cast nephropathy (LCCN), amyloidosis and monoclonal immunoglobulin deposition disease (1). Light chain proximal tubulopathy (LCPT) is a rarely reported entity that classically manifests as acquired Fanconi syndrome and is characterized by the presence of $\kappa$-restricted crystals or phagolysosomes in the proximal tubular epithelia (2). We herein report an unusual case of acquired Fanconi syndrome and acute kidney injury due to combined LCCN and LCPT in which the patient had cytoplasmic fibrillary inclusions of $\lambda$ light chain restriction in the proximal tubular epithelia.

\section{Case Report}

A 68-year-old man was admitted to our hospital for prote- inuria and acute kidney injury. The patient's past medical history and family history showed no previous kidney disease. A urinalysis revealed severe generalized aminoaciduria, proteinuria and glycosuria. The level of urinary protein excretion was $3.96 \mathrm{~g} / \mathrm{day}$ (urine volume, $1,850 \mathrm{~mL}$ ), $93 \%$ of which comprised low-molecular-weight protein. The level of urine $\alpha 1$ microglobulin was $166 \mathrm{mg} / \mathrm{L}$ (reference range: 0 $12 \mathrm{mg} / \mathrm{L}$ ) and the level of urine N-acetyl- $\beta$-Dglucosaminidase (NAG) was $93 \mathrm{U} / \mathrm{L}$ (reference range: 0-21 $\mathrm{U} / \mathrm{L}$ ). The urinary potassium level was $22 \mathrm{mmol} / \mathrm{L}$, while the serum potassium level was $3.3 \mathrm{mmol} / \mathrm{L}$. The serum glucose level was $82.8 \mathrm{mg} / \mathrm{dL}$ (reference range: $70-110 \mathrm{mg} / \mathrm{dL}$ ). The serum phosphate and serum uric acid levels were decreased at $2.78 \mathrm{mg} / \mathrm{dL}$ (reference range: $2.9-5.0 \mathrm{mg} / \mathrm{dL}$ ) and $2.25 \mathrm{mg} / \mathrm{dL}$ (reference range: $2.5-7.0 \mathrm{mg} / \mathrm{dL}$ ), respectively, with a creatinine level of $1.8 \mathrm{mg} / \mathrm{dL}$ (reference range: 0.5 $1.5 \mathrm{mg} / \mathrm{dL}$ ). Serum and urine immunofixation electrophoresis revealed monoclonal $\lambda$ free light chains. A bone marrow aspiration smear showed that $59 \%$ of the cells were naive

Renal Division, Department of Medicine, Peking University First Hospital; Institute of Nephrology, Peking University; Key Laboratory of Renal Diseases, Ministry of Health of China, China

Received for publication April 22, 2013; Accepted for publication August 15, 2013

Correspondence to Dr. Su-Xia Wang, suxiawang@bjmu.edu.cn 

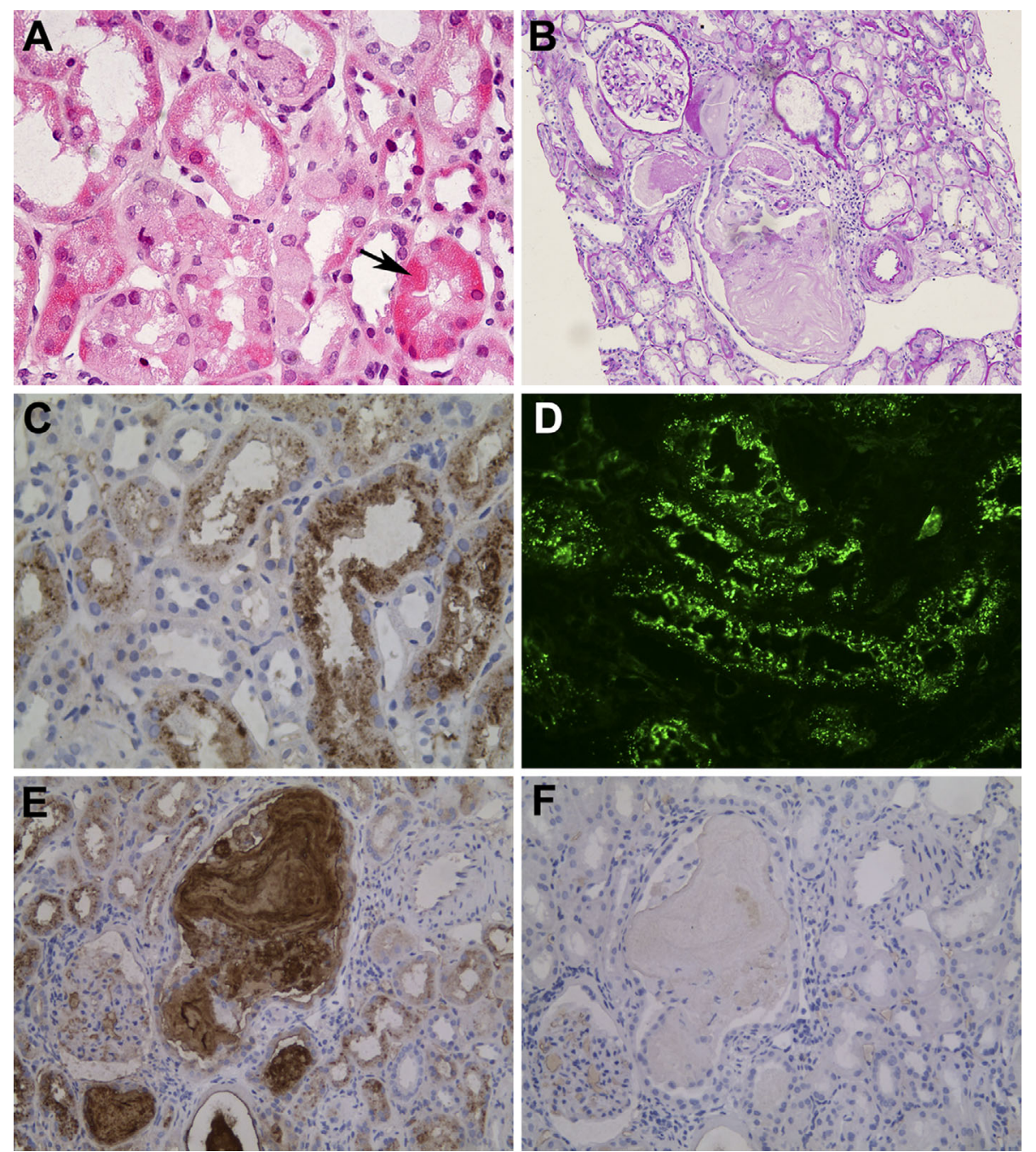

Figure 1. Histological morphology and immunohistochemical staining for light chains. A, The hypertrophied proximal tubules exhibited strongly essinophilic cytoplasm (arrow), some of which showed a loss of brush borders (Hematoxylin and Eosin staining, original magnification $\times 200)$. B, The distal tubular lumen was filled with casts exhibiting a fractured appearance surrounded by mononuclear cells (PAS, original magnification $\times \mathbf{1 0 0})$. $C$, Positive staining for $\lambda$ light chains in the proximal tubular cytoplasm on immunohistochemistry (original magnification $\times \mathbf{2 0 0}$ ). $\mathrm{D}$, Strong positive staining for $\lambda$ light chains in the proximal tubular cytoplasm on immunofluorescence of deparaffinized, protease-treated renal tissue (original magnification $\times \mathbf{2 0 0}$ ). Immunohistochemistry showed that the casts in the distal tubules were positively labeled by $\lambda$ light chains $(E)$ and negatively labeled by $\kappa$ light chains $(\mathbf{F})$ (original magnification $\times \mathbf{2 0 0})$.

plasma cells. The bone marrow tissue contained only a few plasma cells, with a predominant $\lambda$ type on immunohistochemistry. An X-ray examination detected numerous lytic lesions in the calvarium. Multiple myeloma (free $\lambda$ light chain type) was diagnosed.

A renal biopsy showed no significant changes in the glomeruli on light microscopy. The proximal tubular epithelia displayed diffusely degenerative changes, including strongly eosinophilic cytoplasm (Fig. 1A). The distal tubules contained several casts with a fractured appearance surrounded by mononuclear cells (Fig. 1B). The interstitium demonstrated mild infiltration of lymphocytes and monocytes with focal tubular atrophy and interstitial fibrosis. Immunohistochemistry for light chains demonstrated positive staining for $\lambda$ light chains in the tubular cytoplasm (Fig. 1C) and casts (Fig. 1E), while staining for $\kappa$ light chains was essentially negative (Fig. 1F). Positive staining for $\lambda$ light chains in the tubular casts with weak staining in the cytoplasm of the tubular epithelial cells was identified on direct immunofluorescence (IF) of frozen sections, while staining for $\kappa$ light chains was essentially negative. IF of deparaffinized, protease-treated renal tissue sections revealed strong positive staining of $\lambda$ light chain restriction in both the casts and tubular cytoplasm (Fig. 1D).

Electron microscopy showed prominent phagolysosomes (Fig. 2A) and numerous irregularly shaped inclusions in the cytoplasm of the proximal tubules. The inclusions consisted of a fibrillary structure without crystals (Fig. 2B). On immu- 

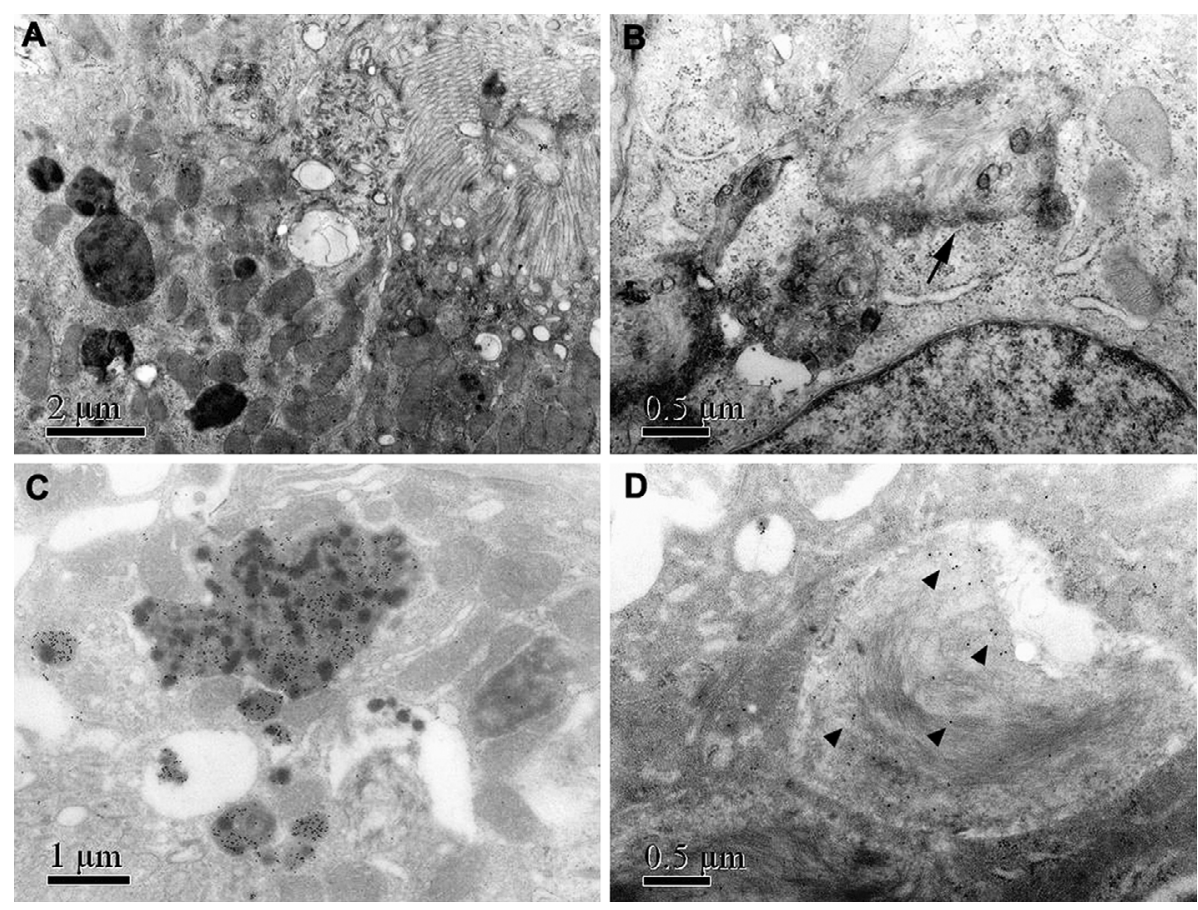

Figure 2. Electron micrographs and immunogold staining for light chains. The proximal tubular cytoplasm was filled with phagolysosomes $(A$, original magnification $\times 15,000)$ and irregularly shaped inclusions (arrow) with a fibrillary matrix $(B$, original magnification $\times 40,000)$. Both the lysosomal content and fibrillary inclusions (arrowheads) were labeled by anti- $\lambda$ light chain antibodies conjugated with colloid gold particles, diameter: $15 \mathrm{~nm},(\mathrm{C}$, original magnification $\times 25,000$; $D$, original magnification $\times \mathbf{4 0 , 0 0 0}$ ).

nogold labeling, the lysosomal content and tubular casts were strongly labeled with $\lambda$ light chain restriction (Fig. 2C), while the inclusions with a fibrillary matrix exhibited lesser labeling for $\lambda$ light chains (Fig. 2D). Labeling for $\kappa$ light chains was negative. Hence, LCPT coexisting with LCCN of $\lambda$ light chain restriction was diagnosed.

Clinical follow-up: The patient was treated with bortezomib and dexamethasone (BD) in addition to thalidomide. After the second cycle of therapy (two months after the renal biopsy), monoclonal proteins were negative in the serum and urine when analyzed using immunofixation electrophoresis. The serum free light chain value was not available due to the lack of free light chain testing at our institute. One year after the renal biopsy, the patient's serum creatinine level was stable at $1.3 \mathrm{mg} / \mathrm{dL}$. Tests for urine protein and urine glucose were negative. The levels of serum uric acid and serum phosphate were normal.

\section{Discussion}

We herein presented the case of a patient with multiple myeloma who developed Fanconi syndrome and acute kidney injury. A renal biopsy showed acute tubular injury with the coexistence of LCCN and LCPT confirmed by the identification of the localization of $\lambda$ light chain restriction in phagolysosomes and cytoplasmic fibrillary inclusions using the immunogold labeling method.

LCPT is a rarely reported entity that usually manifests as acquired Fanconi syndrome. It has been demonstrated that pathologic light chains exhibit resistance to proteolysis by the tubular lysosomal enzymes pepsin and cathepsin B and accumulate within lysosomes where they undergo degradation (3). Reported cases of LCPT mostly involved $\kappa$ light chain restriction with cytoplasmic crystals, whereas LCPT of the $\lambda$ type is rarely described and remains underdiagnosed (4). There are few studies of cases of LCPT without crystal formation, except for Larsen et al., who reported the largest case series of LCPT. In that study, there were 13 cases of LCPT, only three of which involved tubular cytoplasmic crystals. Meanwhile, the majority of cases were characterized by the presence of prominent phagolysosomes or irregularly shaped inclusions in the cytoplasm of the proximal tubules, 10 of which involved $\lambda$ light chain restriction (4). Recently, Taneda et al. (5) reported a patient with proximal tubulopathy induced by the accumulation of fibrillary cytoplasmic inclusions; however, that case involved $\kappa$ light chain restriction.

The light microscopic findings of LCPT are subtle, usually revealing nonspecific acute tubular injury with tubular epithelial degenerative changes. Proximal tubules packed with intracytoplasmic inclusions or crystals show brightly fuchsinophilic staining on trichrome stains or strongly eosinophilic cytoplasm on Hematoxylin and Eosin staining (4). Immunolabeling for monotypic light chains within the cytoplasm of the proximal tubules is an important clue for LCPT. IF of deparaffinized, protease-treated renal tissue 
is more sensitive than direct IF of frozen tissue in detecting light chain proximal tubulopathy (6). In the present case, $\lambda$ light chain immunostaining was weakly positive on direct IF of frozen tissue and strongly positive on IF of deparaffinized, protease-treated renal tissue.

Performing careful ultrastructural examinations of the tubular epithelia using electron microscopy is essential for diagnosing LCPT. In the present case, tubular cytoplasmic lysosomes and fibrillary inclusions were identified on ultrastructural observation and labeled with $\lambda$ light chain restriction using immune electron microscopy. In our opinion, as a result of the transformed structure of the light chains due to incomplete proteolysis by the lysosomal enzymes, the density of labeling of the fibrillary inclusions was lesser than that of the phagolysosomes.

Renal involvement of the PCD usually presents as one pattern of renal pathology, although the occurrence of two or more patterns in the same patient has also been previously described. Patients presenting with both typical myeloma cast nephropathy and full-blown Fanconi syndrome with crystals in the proximal tubule cells have been previously reported in the setting of high-mass myeloma (2). It has been postulated that the overproduction of light chains exceeds the endocytotic capacity of the proximal tubules and that light chains are delivered to the distal tubules where they interact with Tamm-Horsfall proteins and form casts. In our case, the patient had high-mass myeloma; however, $\lambda$ light chains were involved, which is quite different from that observed in the cases reported by Messiaen and associates (2).

This is an unusual case involving the coexistence of LCCN and LCPT in which the patient manifested acquired
Fanconi syndrome characterized by the presence of $\lambda$ restricted fibrillary inclusions in the proximal tubular cytoplasm. Ultrastructural investigations and immune electron microscopy are thus considered to be special sensitive methods for detecting and diagnosing LCPT.

The authors state that they have no Conflict of Interest (COI).

\section{Acknowledgement}

We thank Prof. Yan-Ling Yang, Pediatric Department, Peking University First Hospital for her assistance with the urinary amino acid analysis.

\section{References}

1. Herrera GA. Renal lesions associated with plasma cell dyscrasias: practical approach to diagnosis, new concepts, and challenges. Arch Pathol Lab Med 133: 249-267, 2009.

2. Messiaen T, Deret S, Mougenot B, et al. Adult Fanconi syndrome secondary to light chain gammopathy. Clinicopathologic heterogeneity and unusual features in 11 patients. Medicine (Baltimore) 79: 135-154, 2000.

3. Leboulleux M, Lelongt B, Mougenot B, et al. Protease resistance and binding of Ig light chains in myeloma-associated tubulopathies. Kidney Int 48: 72-79, 1995.

4. Larsen CP, Bell JM, Harris AA, Messias NC, Wang YH, Walker PD. The morphologic spectrum and clinical significance of light chain proximal tubulopathy with and without crystal formation. Mod Pathol 24: 1462-1469, 2011.

5. Taneda S, Honda K, Horita S, et al. Proximal tubule cytoplasmic fibrillary inclusions following kidney transplantation in a patient with a paraproteinemia. Am J Kidney Dis 53: 715-718, 2009.

6. Nasr SH, Galgano SJ, Markowitz GS, Stokes MB, D’Agati VD. Immunofluorescence on pronase-digested paraffin sections: a valuable salvage technique for renal biopsies. Kidney Int 70: 21482151, 2006.

(C) 2014 The Japanese Society of Internal Medicine http://www.naika.or.jp/imonline/index.html 